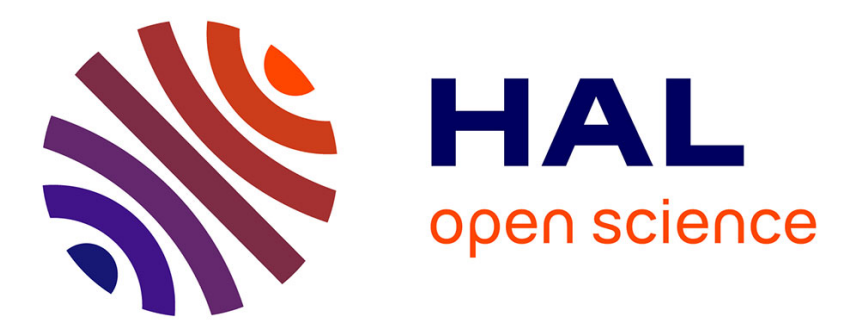

\title{
La génétique de l'hôte influe sur les écosystèmes microbiens par l'intermédiaire du système immunitaire de l'hôte
}

Hela El Kafsy, Guy Gorochov, Martin Larsen

\section{- To cite this version:}

Hela El Kafsy, Guy Gorochov, Martin Larsen. La génétique de l'hôte influe sur les écosystèmes microbiens par l'intermédiaire du système immunitaire de l'hôte. Biologie Aujourd'hui, 2017, 211 (1), pp.39 - 49. 10.1051/jbio/2017010 . hal-01787064

\section{HAL Id: hal-01787064 https: / hal.sorbonne-universite.fr/hal-01787064}

Submitted on 7 May 2018

HAL is a multi-disciplinary open access archive for the deposit and dissemination of scientific research documents, whether they are published or not. The documents may come from teaching and research institutions in France or abroad, or from public or private research centers.
L'archive ouverte pluridisciplinaire $\mathbf{H A L}$, est destinée au dépôt et à la diffusion de documents scientifiques de niveau recherche, publiés ou non, émanant des établissements d'enseignement et de recherche français ou étrangers, des laboratoires publics ou privés. 


\title{
La génétique de l'hôte influe sur les écosystèmes microbiens par l'intermédiaire du système immunitaire de l'hôte
}

\author{
Hela El Kafsy ${ }^{1}$, Guy Gorochov ${ }^{1,2}$ et Martin Larsen ${ }^{1,2}$ \\ 1 Centre d'Immunologie et des Maladies Infectieuses (CIMI-Paris UMRS1135), Sorbonne Universités, \\ UPMC Univ Paris 06, INSERM, Paris, France \\ 2 Département d'Immunologie, AP-HP, Groupement Hospitalier Pitié - Salpêtrière, Paris, France
}

Auteur correspondant : guy.gorochov@inserm.fr

\begin{abstract}
Résumé - L'évolution génétique des organismes multicellulaires a eu lieu en réponse aux agressions de l'environnement, notamment la compétition pour les nutriments, les changements climatiques, les facteurs de stress physiques et chimiques et les pathogènes. Mais l'adaptation d'un organisme dépend d'une part de l'efficacité de ses défenses, mais aussi de sa capacité à tirer avantage de symbioses. En effet les microbes n'engendrent pas seulement des pathogénies, mais permettent aussi l'assimilation efficace de nutriments à partir de régimes non biodégradables par l'hôte lui-même. De plus les microbes jouent un rôle important dans le développement de l'immunité de l'hôte. Nous allons passer en revue ici les associations entre certains gènes spécifiques de l'hôte et la variabilité de composition des microbiotes en les comparant avec les interactions entre microbes et système immunitaire de l'hôte. Les études récentes d'associations pangénomiques révèlent que la symbiose entre hôte et microbiotes est le résultat pointu d'une co-évolution génétique. Par ailleurs un sous-groupe de microbes isolé des microbiotes murin et humain a été identifié pour son interaction avec les immunités humorale et cellulaire. De manière remarquable, les microbes qui réagissent à la fois avec la génétique de l'hôte et avec son immunité sont apparentés sur le plan taxinomique. Les plus performants de ces genres bactériens sur ces deux aspects sont Bifidobacterium, Lactobacillus et Akkermansia. Nous en concluons que les thérapies futures visant les microbiotes dans le cadre de maladies inflammatoires chroniques doivent considérer simultanément les caractéristiques immunes et génétiques de l'hôte liées à l'homéostasie des microbiotes.
\end{abstract}

Mots clés : Maladies inflammatoires chroniques / commensaux / immunogénétique / microbes pathogènes / microbiome

Abstract - Genetic evolution of multicellular organisms occurred as a response to environmental challenges, in particular competition for nutrients, climatic change, physical and chemical stressors and pathogens. However organism fitness depends on both the efficiency of its defences and its capacities for benefiting from its symbiotic organisms. Indeed microbes not only engender pathogenies, but enable efficient uptake of host non-self biodegradable nutriments. Furthermore, microbes play an important role in the development of host immunity. We shall review here the associations between some specific genes of the host, microbiota and the immune system. Recent genomewide association studies disclose that symbiosis between host and microbiota results from a stringent genetic co-evolution. On the other hand, a microbe subset isolated from murine and human microbiotes has been identified on the basis of its interaction with both the host genetics and immunity. Remarkably, microbes which have two such connections are taxonomically related. The best performing bacterial genuses in these two perspectives are Bifidobacterium, Lactobacillus and Akkermansia. We conclude that future therapies targeting microbiota within the framework of chronic inflammatory diseases must consider together host immune and genetic characters associated with microbiota homeostasis. 


\section{Abréviations}

$\begin{array}{ll}\text { AHR : } & \text { Aryl Hydrocarbon Receptor } \\ \text { Bmal1 : } & \begin{array}{l}\text { protéine-1 AHR translocator-like nucléaire } \\ \text { CARD9 : }\end{array} \\ & \begin{array}{l}\text { membre } 9 \text { de la famille du domaine } \\ \text { de recrutement des caspases }\end{array} \\ \text { FLG : } & \text { filaggrine } \\ \text { GWAS : } & \text { Genome Wide Association Studies } \\ \text { IBD : } & \text { Inflammatory Bowel Disease } \\ \text { iTreg : } & \text { cellules régulatrices T inductibles } \\ \text { KO : } & \text { Knock Out } \\ \text { LCT : } & \text { gène de la lactase } \\ \text { QTL : } & \text { Quantitative Trait Loci } \\ \text { RORE : } & \text { acide rétinoïque } \\ \text { SIgA : } & \text { IgA sécrétoires } \\ \text { SNP : } & \text { Single Nucleotide Polymorphism } \\ \text { TMF : } & \text { transplantation de microbiote fécal }\end{array}$

\section{Introduction}

Les organismes multicellulaires ont évolué dans des environnements complexes occupés par des écosystèmes microbiens divers. Cette co-évolution, menant au développement de relations symbiotiques entre les microbes et les organismes plus complexes, a été étroitement régulée par ces derniers. L'hôte a développé des stratégies antimicrobiennes (par exemple la promotion de fonctions de barrières étroitement contrôlées), mais aussi promicrobiennes (sécrétion de substances nourrissantes, telles que le mucus). L'immunité de l'hôte elle-même n'est pas seulement partie prenante de la défense de l'hôte, mais aussi intimement associée à la symbiose avec les microbes. Le système immunitaire peut donc exercer simultanément des pressions anti- mais aussi pro-microbiennes. Les symbiontes activent à la fois l'immunité innée et adaptative de l'hôte, ce qui modifie non seulement l'immunité dirigée contre les symbiontes mais aussi celle qui s'adresse aux pathogènes. L'acquisition d'une complexité immune trans-spécifique (c'est-à-dire l'acquisition de l'immunité adaptative) est associée positivement à la diversité du microbiote implanté dans l'hôte, ce qui suggère que l'immunité évoluée permet le contrôle d'écosystèmes microbiens hautement divers, exploitant ainsi leur potentiel métabolique. Les symbiontes protègent également leur hôte des pathogènes grâce à la compétition pour les sources d'énergie et la sécrétion de substances antimicrobiennes (Kostic et al., 2013).

Les facteurs environnementaux influencent fortement la composition des microbiotes, ce qui a été démontré de manière répétée. Par exemple les changements de régime (Cotillard et al., 2013), le mode de vie (Claesson et al., 2012) et les migrations (Yatsunenko et al., 2012; Ou et al., 2013) ont une influence importante sur la composition du microbiote intestinal. Toutefois la co-évolution de l'hôte et de son microbiote plaide pour un composant génétique de l'hôte qui régulerait la composition du microbiote à la fois dans l'espèce et entre les espèces. Il est en général difficile de distinguer les effets environnementaux des effets génétiques d'après des caractères biologiques. Chez l'Homme, la meilleure manière de procéder est d'étudier des paires de jumeaux monozygotes et dizygotes, dont on peut considérer qu'ils ont rencontré les mêmes facteurs d'environnement, s'ils ont été élevés ensemble. Et en effet, la première étude conduite sur une petite cohorte de jumeaux (van de Merwe et al., 1983), qui ait décrit la variation génétique de l'hôte humain comme un déterminant de la composition du microbiote intestinal, a suggéré que les jumeaux monozygotes ont des microbiotes plus proches que les jumeaux dizygotes. Cette similitude a ensuite été confirmée sur des cohortes plus importantes à l'aide d'analyses de séquencage actualisées (Goodrich et al., 2014; Lim et al., 2016a). Au niveau de la population, on a récemment démontré qu'une variété de maladies chroniques, telles que la colite inflammatoire (Palm et al., 2014), le diabète (Forslund et al., 2015; Qin et al., 2012) et l'allergie (Hua et al., 2016), qui ont une contribution génétique bien établie, sont également associées à des microbiotes intestinaux dysbiotiques. On ne sait pas encore si la dysbiose observée dans ces pathologies dépend ou non de la génétique de l'hôte.

\section{Héritabilité des microbiotes intestinaux dans des modèles animaux}

Benson et al. (2010) sont les premiers qui ont identifié, dans une lignée de souris issue de croisements entre plusieurs souches, des loci de caractères quantitatifs (Quantitative Trait Loci $=$ QTL) associés à des taxons majeurs de microbes intestinaux. Ils ont démontré que l'abondance d'Helicobacter (Protéobactéries; Epsilonprobactéries), de Lactobacillus (Firmicutes; Bacilli), de Proteobacteria, de Coriobacteriacceae (Actinobacteria; Actinobacteria) et de Lactococcus (Firmicutes; Bacilli) est associée à des QTL localisés essentiellement sur les chromosomes 6, 7 et 10 (Tab. 1) (Benson et al., 2010; Olivares et al., 2015; Hov et al., 2015; Si et al., 2015; Davenport, et al., 2015; Blekhman et al., 2015; Goodrich et al., 2016; Org et al., 2015; Kubinak et al., 2015; Liang et al., 2015; Chaston et al., 2016, Lamas et al., 2016; Lim et al., 2016b). En effet l'abondance des Coriobacteriacceae (Actinobacteria; Actinobacteria) et celle des Lactococus (Firmicutes; Bacilli) est associée à des QTL situés sur des chromosomes en rapport respectivement avec la signalisation du 


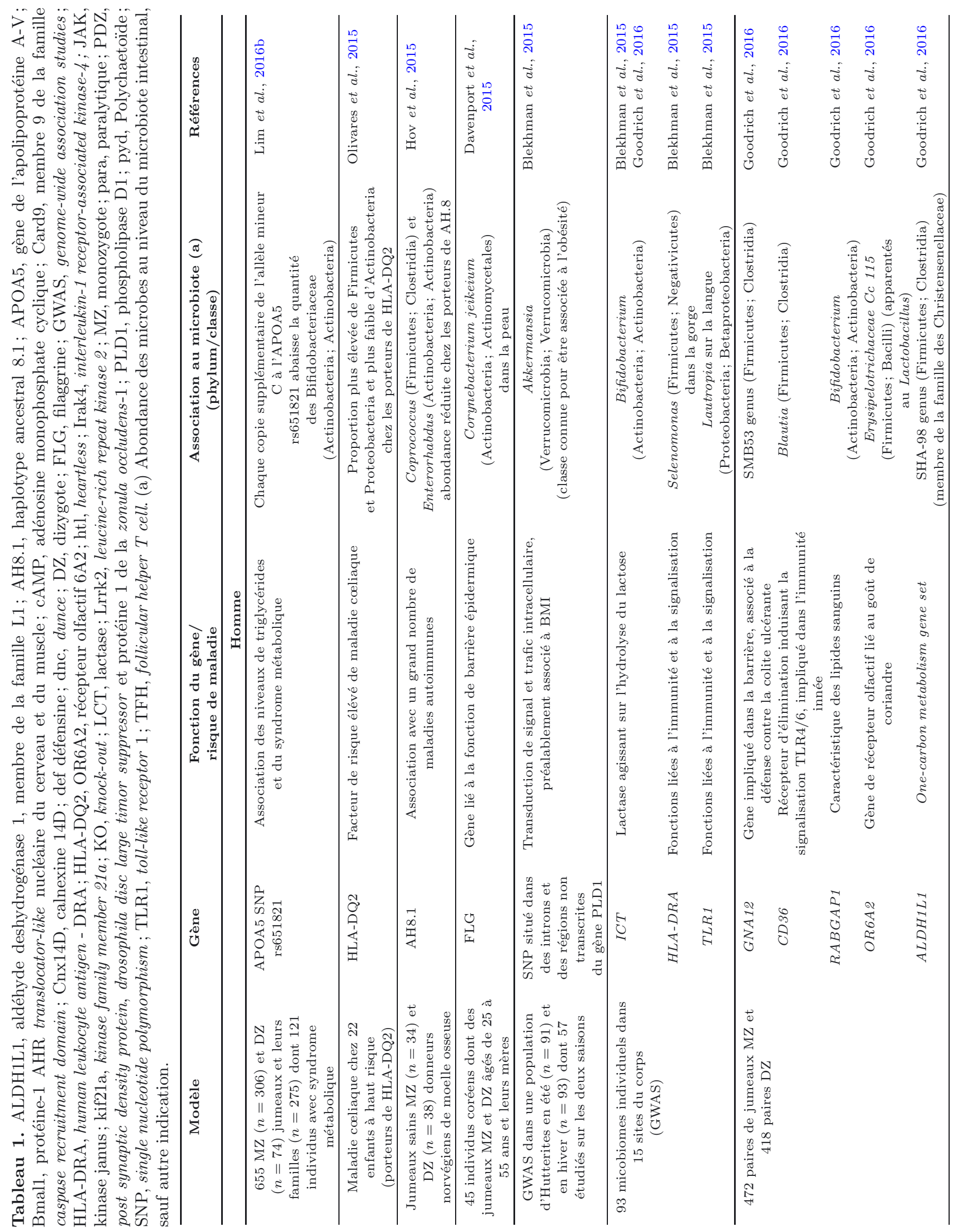




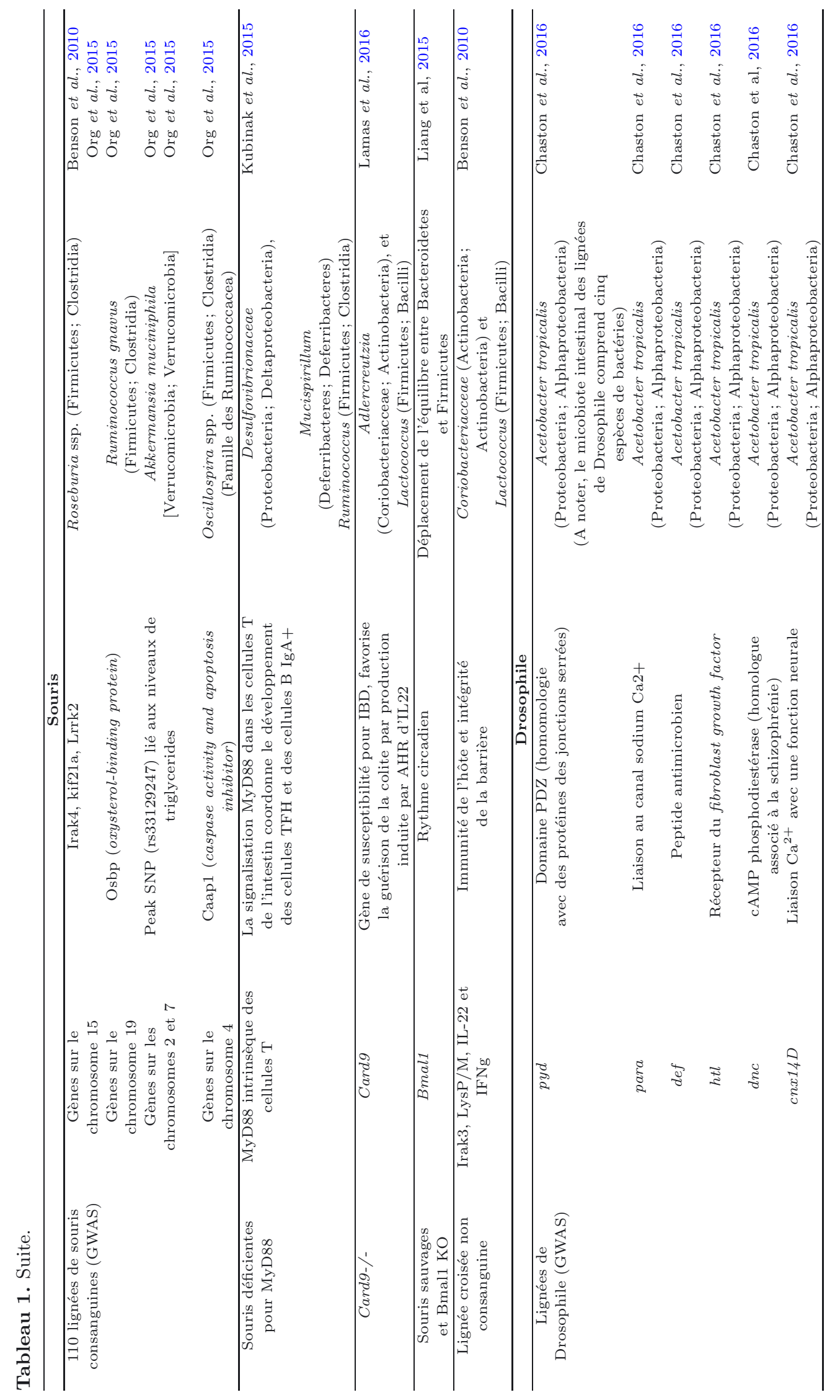


récepteur Toll-like médié par MyD88 et avec la signalisation par le récepteur des cellules $\mathrm{T}$ (kinase 4 et IRAK3, associées au récepteur de l'IL1) ainsi qu'avec des enzymes antibactériennes sécrétées dans les larmes, la salive et le mucus (gènes du lysozyme Lyz1 et Lyz2) et les cytokines proinflammatoires IL22 et IFN. Une étude indépendante (Org et al., 2015) a montré qu'IRAK4 est associée à Roseburia spp. (Firmicutes; Clostridia). MyD88 est localisé en amont de IRAK4 dans la cascade de signalisation. Les mutations de $M y D 88$, qui diminuent l'activité de MyD88, avaient déjà été reliées à un risque accru d'infections bactériennes et les souris KO pour MyD88 présentent des taux diminués d'IgA sécrétoires ( $\operatorname{sIg} \mathrm{A}$ ) et une augmentation de nombreux taxons comportant des membres mucolytiques, en particulier Desulfovibrionaceae (Protéobactéries; Deltaprotéobactéries), Mucispirillum (Deferribacteres; Deferribacteres), et Ruminococcus (Firmicutes; Clostridia) (Tab. 1).

De plus, Org et al. (2015) ont identifié deux gènes qui codent pour l'inhibiteur 1 des activités caspase et apoptotique (Caap1) et pour une protéine de liaison à l'oxystérol $(O s b p)$, gènes qui ont été associés respectivement à une abondance d'Oscillospora spp. (Firmicutes; Bacilli) et de Ruminococcus gnavus (Firmicutes; Clostridia). Un autre membre de la voie caspase est le membre 9 de la famille du domaine de recrutement des caspases (CARD9). Une mutation homozygote de CARD9, qui détermine un codon de terminaison prématurée, a été associée à une susceptibilité à l'infection fongique, probablement causée par le défaut d'une signalisation innée du récepteur dectine-1 (Glocker et al., 2009) reconnaissant un pattern fongique, signalisation également cruciale pour la capture par les cellules $\mathrm{M}^{1}$ des antigènes liés aux sIgA et pour la présentation en aval des antigènes (Rochereau et al., 2013). Par ailleurs CARD9 est associé aux maladies inflammatoires intestinales, vraisemblablement par suite d'une abondance réduite de bactéries comme Lactobacillus (Firmicutes ; Bacilli), capables de métaboliser le tryptophane en agoniste du récepteur des aryl hydrates de carbone (AHR) (Lamas et al., 2016). L'activité AHR stimule la sécrétion intestinale localisée d'IL-22, qui a une influence importante sur la fonction de barrière et sur les mécanismes de défense anti-bactérienne (sécrétion de défensines). Effectivement un traitement par des souches de Lactobacillus guérit les souris colitiques. De plus les souris $C A R D 9^{-} /^{-}$présentent une abondance significativement réduite d'Adlercreutzia

\footnotetext{
${ }^{1}$ Les plaques de Peyer sont des agrégats de follicules lymphoïdes situés à intervalles réguliers dans la partie terminale de l'iléon. Ces follicules sont séparés de la lumière intestinale par des cellules épithéliales particulières, dites cellules M (pour microfold cells).
}

(Actinobacteria; Actinobacteria), un membre de la famille des Coribacteriaceae (Lamas et al., 2016). On l'a vu plus haut, les Coriobacteriaceae ont été associées à un QTL situé sur le chromosome 10 de la souris, qui comporte des gènes codant pour l'IL-22 (Benson et al., 2010). Etant donné que seul CARD9 (mais aucun des QTL identifiés dans l'étude précédente) est situé sur le chromosome murin 2, il est possible que l'association entre Adlercreutzia et CARD9 soit un effet indirect de l'influence de CARD9 sur la sécrétion d'IL-22 médiée par AHR.

La protéine 1 (Bmal1) nucléaire, cérébrale et musculaire, AHR translocatrice-like, est un facteur de transcription impliqué dans la régulation du rythme circadien, lui-même régulé au niveau de la transcription par un site situé dans le promoteur de Bmal1, qui lie un élément de réponse à un récepteur orphelin apparenté à l'acide rétinoïque (RORE). L'un des facteurs de transcription ROR, qui lient RORE, est $\operatorname{ROR} \gamma$, qui régule étroitement la différenciation des cellules Th17 et le maintien de l'équilibre entre cellules régulatrices $\mathrm{T}$ inductibles (iTreg) et cellules Th17. On a récemment démontré que l'équilibre entre les microbes intestinaux des phylums Bacteroidetes et Firmicutes est associé au rythme circadien avec un pic de Bacteroidetes après plusieurs heures de phase sombre et un pic de Firmicutes au début de la phase éclairée (Liang et al., 2015). Les souris knock out $\mathrm{Bmal1}^{-/-}$perdent cette alternance jour/nuit de leur microbiote intestinal. Enfin, deux haplotypes de Bmal1 chez l'Homme sont associés au diabète de type 2 (Woon et al., 2007), maladie associée à une dysbiose du microbiote intestinal (Qin et al., 2012; Forslund et al., 2015).

La barrière intestinale sépare physiquement le microbiote intestinal de l'hôte. Elle sert ainsi de première ligne de défense. La seconde ligne inclut les fonctions immunes innées, comme les peptides antimicrobiens (les défensines), qui sont sécrétés par les cellules de la barrière en réponse à la perception par l'hôte des microbes qui menacent de se transloquer au travers de la barrière et de l'envahir. Du point de vue évolutif, ces mécanismes de défense sont apparus très tôt. Un certain nombre de gènes régulant ces voies défensives présentent un polymorphisme, même chez des organismes moins complexes, tels que Arabidopsis (Horton et al., 2014) et la drosophile (Chaston et al., 2016). Effectivement, le polymorphisme de gènes comme polychaetoïde (pyd) et défensine (def chez la drosophile) a été relié à une dysbiose du microbiote intestinal, caractérisée par une variation dans l'abondance d'Acetobacter tropicalis (Proteobacteria; Alphaproteobacteria) (Tab. 1). Il faut noter que le gène pyd est l'homologue de gènes humains impliqués dans la régulation des jonctions 
serrées, qui contrôlent la perméabilité de la barrière intestinale.

Les études sur des modèles animaux portant sur l'association entre le polyporphisme génique de l'hôte et son microbiote intestinal mettent en lumière l'importance des défenses de l'hôte dans la régulation de l'homéostasie des microbiotes. Les études animales sont particulièrement bien adaptées à ce type d'analyse, car les causes d'erreur, tels que le régime et l'environnement, peuvent être harmonisées et parce que l'effet de gènes identifiés peut être testé dans des modèles Knock Out. La diversité génétique des hôtes peut notamment être réduite grâce à ces modèles. $A$ contrario dans les études humaines, les facteurs d'erreur ajoutent une variance significative à associer à la variation génétique de la composition du microbiote. Les études classiques d'associations pangénomiques (Genome Wide Association Studies = GWAS), portant sur une quantité limitée de traits phénotypiques, compensent cette variation en augmentant la taille de la cohorte. Toutefois, quand ces traits phénotypiques sont les quantités relatives de centaines de microbes, les comparaisons multiples outrepassent significativement les capacités statistiques, si bien qu'il faut travailler sur des cohortes extrêmement importantes. Dans le chapitre suivant nous discuterons les associations entre gènes humains et abondances microbiennes. Vu la puissance statistique de ces analyses, il est probable que nous n'observons encore que le sommet de l'iceberg. Des associations spécifiques de maladies, un peu plus faibles mais néanmoins significatives, pourraient émerger de futures études plus importantes ou mieux ciblées.

\section{Héritabilité humaine des microbiotes}

La première GWAS humaine, qui ait considéré la composition des microbiotes de différents sites du corps comme un trait phénotypique associatif, comprenait 93 individus du projet « microbiome humain » (Blekhman et al., 2016). Pour gagner de la puissance statistique, les auteurs ont réalisé une analyse de principaux composants à partir des données des microbiotes stratifiés par sites et ont utilisé le «top 5 » des principaux composants de ces analyses comme caractères phénotypiques. Plusieurs voies liées à la défense de l'hôte ont été identifiées par cette approche; elles comprennent en particulier la leptine, JAK/STAT, la signalisation par les chimiokines et par les récepteurs reconnaissant des motifs. Elles étaient toutes associées à la combinaison microbiotique du nez, de la gorge et de la peau. De manière similaire, deux polymorphismes de nucléotides isolés ( $\mathrm{SNP}=$ Single Nucleotide polymorphisms) dans l'antigène leukocytaire humain DRA et dans le récepteur 1 Toll-like ont pu être associés respectivement à une abondance de Selenomonas dérivés de la gorge et de Lautropia dérivés du dessus de la langue (Tab. 1).

Du Bifidobacterium obtenu de l'intestin a été relié à un SNP du gène de la lactase $(L C T)$, qui code pour l'enzyme pivot du clivage du lactose des laitages. Notons que le polymorphisme de $L C T$ est associé à la tolérance au lactose. De plus les individus qui ont un gène $L C T$ fonctionnel ont des niveaux de Bifidobacterium réduits, probablement parce que l'hôte dégradant le lactose, le Bifidobacterium y est privé d'une source d'énergie importante. Il est intéressant que le lien entre $L C T$ et Bifidobacterium ait également été observé dans les populations huttérites ${ }^{2}$ sans toutefois atteindre une signification statistique (Davenport et al., 2015), et confirmé dans la plus grande étude à ce jour (Goodrich et al., 2016), qui inclut 2139 individus, parmi lesquels 890 paires de jumeaux monozygotes et dizygotes. Cette dernière étude a même identifié les gènes GNA12 et CD36, qui sont respectivement impliqués dans la barrière de défense et l'immunité innée. Les polymorphismes de GNA12 et CD36 sont associés respectivement à une abondance de SMB53 (Firmicutes; Clostridia) et de Blautia (Firmicutes; Clostridia). Dans une étude indépendante, Si et al. (2015) ont identifié un gène apparenté à la fonction de barrière de l'épithélium, celui de la filaggrine (FLG), qui est associé à une abondance de Corynebacterium jeikeium (Actinobacteria; Actinobacteria). Au final, les haplotypes du gène haplotype ancestral 8.1 (AH8.1) associés à un grand nombre de maladies auto-immunes humaines, de même qu'un polymorphisme du gène $H L A-D Q 2$ lié à un risque génétique important de maladie coeliaque, sont rattachés à la composition microbienne intestinale (Tab. 1).

Au-delà des gènes de l'hôte liés à l'immunité, un certain nombre de gènes impliqués dans le métabolisme de l'hôte ont été découverts. Deux d'entre eux, déterminant des caractères lipidiques sanguins (apolipoprotéine A-V et protéine 1 d'activation de la Rab GTPase), sont associés à la quantité de Bifidobacterium (Actinobacteria; Actinobacteria) (Goodrich et al., 2016). Deux gènes encore, en cause dans la perception du goût des lipides et le métabolisme du carbone (OR6A2 et ALDH1L1), sont également identifiés pour leur association avec l'abondance respective d'Erysipelotrichaceae Cc 115 (Firmicutes; Bacilli) et de SHA98 (Firmicutes; Clostridia).

\footnotetext{
${ }^{2}$ Groupe ethnoreligieux chrétien implanté au Canada, conservant des mœurs pastorales anciennes.
} 


\section{Dialogue entre le microbiote intestinal et l'immunité cellulaire}

Les microbes jouent un rôle important dans le développement et la maturation de l'immunité de l'hôte. Effectivement, les souris axéniques présentent un système immunitaire sévèrement déficient, dans lequel le développement des plaques de Peyer et des centres germinatifs de l'intestin grêle est affecté. En l'absence des sites spécialisés dans la différenciation et la maturation des lymphocytes $\mathrm{T}$ et $\mathrm{B}$, les souris axéniques ont des niveaux réduits de cellules $\mathrm{T}$ $\mathrm{CD}^{+}$et $\mathrm{CD}^{+}$, y compris des iTreg. Les cellules Th17 présentent une dichotomie entre des niveaux diminués dans l'intestin grêle et augmentés dans le côlon. De plus, les souris axéniques ont des niveaux complètement affaissés de sIgA (Round et al., 2009).

Plus précisément, les cellules Th17 peuvent être induites par les espèces mucolytiques du microbiote intestinal, par exemple les bactéries filamenteuses segmentées (SFB), Citrobacter rodentium et Escherichia coli, mais aussi par un consortium de 20 bactéries à caractéristiques mucolytiques, isolées d'un patient affecté de colite ulcéreuse (Atarashi et al., 2015). De même, l'induction et la maturation des iTreg intestinales sont affectées par la présence de nombreuses espèces du genre Clostridium ou du genre Lactobacillum (Lavasani et al., 2010; Atarashi et al., 2015). Un traitement probiotique de souris à l'aide de ces souches agit sur le développement et la maturation de ces cellules. Mieux même, le polysaccaride A libéré par plusieurs espèces du genre Bacteroides est capable d'induire la sécrétion d'IL10 par les cellules dendritiques, ce qui peut promouvoir la différenciation de iTreg (Ochoa-Reparaz et al., 2010; Round \& Mazmanian, 2010).

\section{Dialogue entre le microbiote intestinal et l'immunité humorale (secretory $\lg \mathrm{A}$ )}

Bien que les microbes colonisent tous les habitats accessibles, y compris la peau, la bouche, le nez, le vagin et même les poumons, presque toutes nos connaissances sur l'impact de la colonisation microbienne naturelle sur l'immunité de l'hôte sont restreintes à la communauté naturelle la plus dense, le microbiote intestinal. Dans les années récentes, le développement de l'analyse de flux cytométrique appliquée au microbiote intestinal lié aux sIgA (Moor et al., 2016) et des procédures associées (Cullender et al., 2013) a permis une évaluation précise du microbiote intestinal ciblé par les sIgA (D'Auria et al., 2013; Palm et al., 2014; Bunker et al., 2015; Kau et al., 2015; Planer et al., 2016). Toutes ces études décrivent une réponse immune sIgA contre le microbiote intestinal hétérogène, avec d'importantes variations entre individus sains. Ces études s'accordent cependant sur le point suivant : les cibles principales des $\operatorname{sig} \mathrm{A}$ sont trouvées dans les trois phylums des Firmicutes, Proteobacteria, et Actinobacteria, qui sont aussi les plus transmissibles. Les interactions $\operatorname{sIg} \mathrm{A} /$ microbes identifiées dans la littérature sont résumées dans la figure 1 qui met en vedette Bifidobacterium, Lactobacillus, Clostridium, SFB, Escherichia, et Akkermansia comme des cibles spéciales des sIgA. Notons que Lactobacillus, Clostridium, SFB et Escherichia influent toutes sur l'immunité cellulaire, tandis que Bifidobacterium, Lactobacillus, Clostridium et Akkermansia ont été reliés aux polymorphismes des gènes de l'hôte. Bifidobacterium est associé aux gènes de l'hôte impliqués dans des voies de défense et de non défense; Lactobacillus et Clostridium sont rattachés uniquement aux gènes de défense et Akkermansia aux gènes de non défense. Il est curieux que les genres associés à la biologie des cellules $\mathrm{T}$ régulatrices (Lactobacillus et Clostridium) soient les deux seuls également reliés à la fois aux polymorphismes des $\operatorname{sig} \mathrm{A}$ et des gènes de défense de l'hôte (Fig. 1). Au contraire, les cellules Th17 sont associées avec les genres pathobiontes (SFB, E. coli et Citrobacter) cibles des SIgA mais non liés à la génétique de l'hôte. Il est donc tentant de spéculer que la santé de l'hôte a, par l'évolution génétique, accordé la priorité à la régulation de la symbiose entre hôte et microbiote, avant l'élimination du danger extérieur par des réponses proinflammatoires.

Il est intéressant qu'on n'ait trouvé aucune bactérie du phylum Bacteriodetes (soit environ $50 \%$ du microbiote intestinal humain) qui interagisse avec les sIgA dans les conditions non pathologiques, ni qui s'associe aux polymorphismes du génome de l'hôte. Il est possible que la longue adaptation évolutive entre hôtes et bactéries dérivées du genre Bacteroidetes force particulièrement la conservation des gènes associés de l'hôte et par conséquent la rend indétectable par le GWAS. Palm et al. (2014) ont remarqué que les bactéries dérivées des Bacteroidetes de la famille des Prevotellacaceae sont liées par les SIgA chez les souris souffrant de colite, ce qui suggère que des associations délétères entre la génétique de l'hôte et son immunité pourraient être observées dans les études de cohortes de malades. De plus ces auteurs ont démontré que les microbiotes intestinaux ciblés par les sIgA sont colitogènes et provoquent des désordres chez des souris saines transplantées. Finalement, plusieurs gènes de l'immunité ne sont associés aux niveaux de microbes que dans la gorge et sur le dessus de la langue, et non dans l'intestin. Il est par conséquent probable que les travaux futurs portant sur ces parties du corps révèleront beaucoup d'autres interactions entre l'immunité de l'hôte et les microbiotes. 


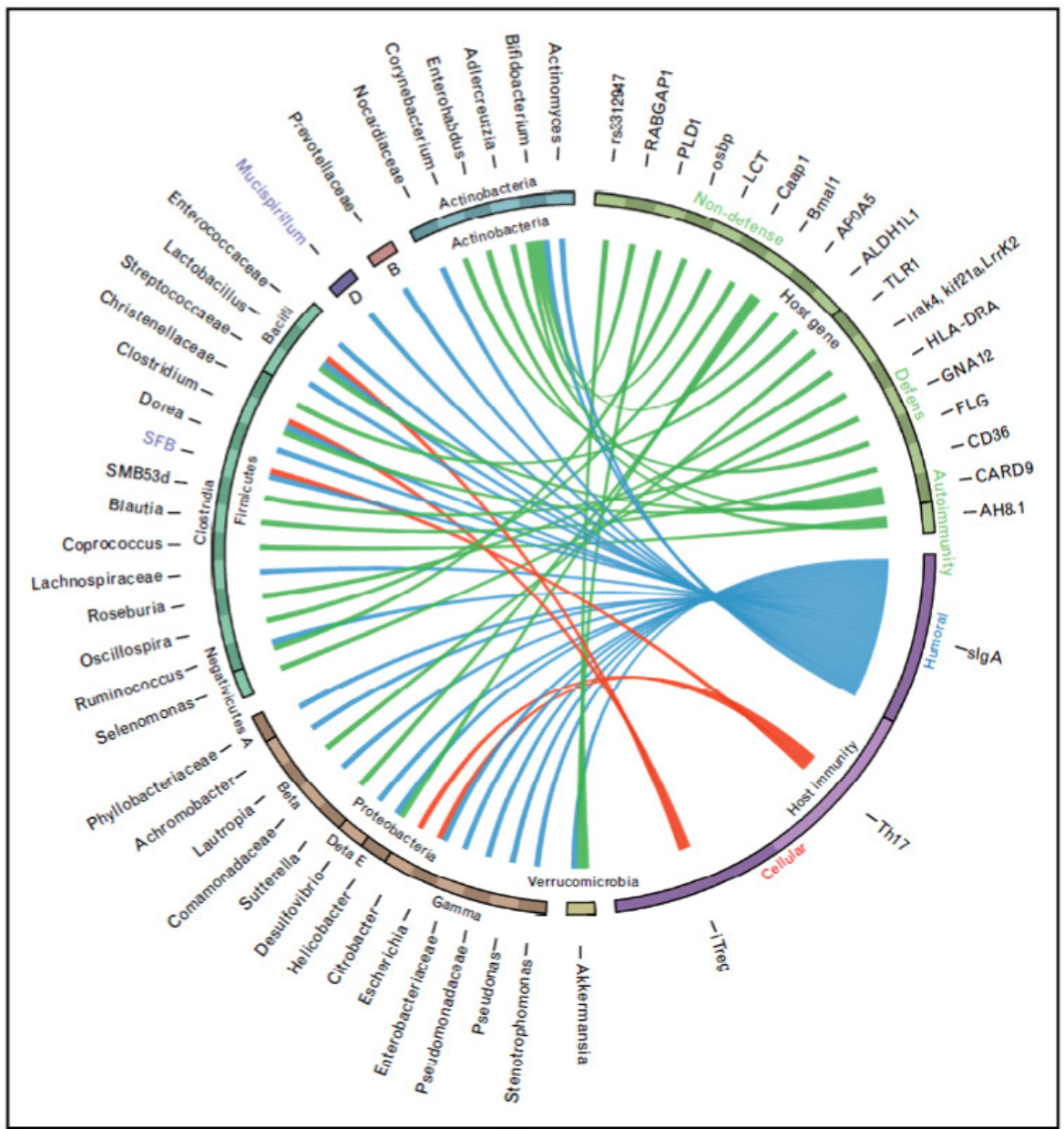

Fig. 1. Le diagramme illustre les associations entre les membres du microbiote et les caractères de l'hôte (génétiques et immunitaires). Les membres du microbiote sont stratifiés selon leur phylum (labels du cercle interne) et leur classe (cercle externe). Nota bene : les phylums B et D correspondent respectivement aux Bacteroidetes et aux Deferribacteres. Chaque membre est identifié du point de vue taxinomique sur le plan de la famille ou du genre (labels extérieurs radiaux). Les gènes de l'hôte sont groupés selon leurs associations à l'autoimmunité, à la défense de l'hôte ou à d'autres fonctions (non défensives), telles qu'elles ont été rapportées. Les caractéristiques immunes de l'hôte appartiennent à l'immunité humorale (SIgA) et cellulaire (Th17 et iTreg). Les associations entre les microbes et les gènes de l'hôte, les cellules immunitaires et/ou les sIgA sont indiquées par les rubans colorés (verts, rouges et bleus, respectivement). Seuls les microbes, dont les associations soit aux gènes de l'hôte (Tab. 1) soit aux caractéristiques immunes sont connues, sont inclus dans le diagramme. Les associations sont établies d'après les études sur l'Homme et la souris. Deux taxons trouvés dans les microbiotes de rongeurs (mais non chez l'Homme) sont colorés en violet. iTreg : cellule T régulatrice inductible; sIgA : $\operatorname{Ig} \mathrm{A}$ sécrétoire.

\section{Conclusion}

Ces dernières années, la transplantation de microbiote fécal (TMF) a été reconnue comme une solution thérapeutique efficace contre les infections à Clostridium difficile et un traitement des maladies chroniques associées à une dysbiose microbiotique intestinale, telles que l'IBD, le diabète de type 2, l'arthrite rhumatoïde, la sclérose multiple et l'asthme. Savoir comment la structure génétique de l'hôte impacte la composition du microbiote sera de la plus haute importance pour comprendre si les TFM peuvent avoir un effet bénéfique à long terme sur les maladies chroniques ou si la génétique de l'hôte peut accentuer un équilibre dysbiosique. Effectivement, une étude cinétique récente a montré que les transplantations de microbiote fécal sont instables. Dans le futur, cette instabilité doit pouvoir être rapportée non seulement à des styles de vie différents entre donneur et receveur mais aussi à des différences génétiques. Selon l'importance de l'influence de la génétique de l'hôte sur la composition 
du microbiote intestinal, la TMF dans le contexte d'une maladie chronique pourrait requérir une harmonisation génétique entre donneur et receveur pour au moins quelques SNP ou mieux une compensation thérapeutique pour des fonctionnalités perdues ou gagnées selon le génotype du patient.

Jusqu'à présent aucune GWAS (Genome-Wide Association Studies) sur des cohortes d'individus souffrant de maladies allergiques n'a été conduite en considérant la composition du microbiote intestinal comme un caractère phénotypique. Pourtant, des GWAS de maladies allergiques ont déjà identifié de nombreux polymorphismes des gènes de l'hôte associés à l'immunité, en accord avec ceux identifiés pour leur liaison à la composition du microbiote intestinal (Portelli et al., 2015). Effectivement, les gènes $H L A$ $D Q$ et $F L G$, qui sont liés à la composition du microbiote (Tab. 1), sont également associés à l'asthme allergique, la dermatite atopique (eczéma) et la rhinite allergique saisonnière. Par ailleurs la maladie allergique biaise la composition du microbiote intestinal. Il est, par conséquent, probable que les travaux futurs identifieront des liens cruciaux entre la composition du microbiote intestinal et la génétique des maladies allergiques de l'hôte. Cela pourrait éclairer les causes sous-jacentes à ces maladies et guider le développement de thérapeutiques spécifiques des circuits de défense, nécessitées par l'incidence croissante des allergies.

Remerciements. Nous remercions L'Institut National de la Santé et de la Recherche Médicale (INSERM), l'Agence Nationale de la Recherche (MetAntibody, ANR14-CE140013) et la Fondation pour l'Aide à la Recherche sur la Sclérose en Plaques (ARSEP).

\section{Conflits d'intérêt : aucun}

\section{Références}

Abrahamsson T.R., Jakobsson H.E., Andersson A.F., Björkstén B., Engstrand L., Jenmalm M.C. (2014) Low gut microbiote diversity in early infancy precedes asthma at school age. Clin Exp Allergy, 44, 842-850.

Angelberger S., Reinisch W., Makristathis A., Lichtenberger C., Dejaco C., Papay P, Novacek G., Trauner M., Loy A., Berry D. (2013) Temporal bacterial community dynamics vary among ulcerative colitis patients after fecal microbiote transplantation. Am J Gastroenterol, 108, 1620-1630.

Atarashi K., Tanoue T., Ando M Kamada N., Nagano Y., Narushima S., Suda W., Imaoka A, Setoyama H., Nagamori T., Ishikawa E., Shima T., Hara T., Kado S., Jinnohara T., Ohno H., Kondo T., Toyooka K., Watanabe E., Yokoyama S., Tokoro S., Mori H.,
Noguchi Y., Morita H., Ivanov I.I., Sugiyama T., Nuñez G., Camp J.G., Hattori M., Umesaki Y., Honda K. (2015) Th17 cell induction by adhesion of microbes to intestinal epithelial cells. Cell, 163, 367-380.

Benson A.K., Kelly S.A., Legge R., Ma F., Low S.J., Kim J., Zhang M., Oh P.L., Nehrenberg D., Hua K., Kachman S.D., Moriyama E.N., Walter J., Peterson D.A., Pomp D. (2010) Individuality in gut microbiota composition is a complex polygenic trait shaped by multiple environmental and host genetic factors. Proc Natl Acad Sci USA, 107, 18933-18938.

Blekhman R., Goodrich J.K., Huang K., Sun Q., Bukowski R., Bell J.T., Spector T.D., Keinan A., Ley R.E., Gevers D., Clark A.G. (2015) Host genetic variation impacts microbiome composition across human body sites. Genome Biol, 16, 191.

Bunker J.J., Flynn T.M., Koval J.C., Shaw D.G., Meisel M., McDonald B.D., Ishizuka I.E., Dent A.L., Wilson P.C., Jabri B., Antonopoulos D.A., Bendelac A. (2015) Innate and adaptive humoral responses coat distinct commensal bacteria with immunoglobulin A. Immunity, 43, 541-553.

Chaston J.M., Dobson A.J., Newell P.D., Douglas A.E. (2016) Host genetic control of the microbiota mediates the drosophila nutritional phenotype. Appl Environ Microbiol, 82, 671-679.

Claesson M.J., Jeffery I.B., Conde S., Power S.E., O'Connor E.M., Cusack S., Harris HM, Coakley M., Lakshminarayanan B., O'Sullivan O., Fitzgerald G.F., Deane J., O'Connor M., Harnedy N., O'Connor K., O'Mahony D., van Sinderen D., Wallace M., Brennan L., Stanton C., Marchesi J.R., Fitzgerald A.P., Shanahan F., Hill C., Ross RP, O'Toole P.W. (2012) Gut microbiota composition correlates with diet and health in the elderly. Nature, 488, 178-184.

Cotillard A., Kennedy S.P., Kong L.C., Prifti E., Pons N., Le Chatelier E., Almeida M, Quinquis B., Levenez F., Galleron N., Gougis S., Rizkalla S., Batto J.M., Renault P; A.N.R MicroObes consortium, Doré J., Zucker J.D., Clément K., Ehrlich S.D. (2013) Dietary intervention impact on gut microbial gene richness. Nature, 500, 585-588.

Cullender T.C., Chassaing B., Janzon A., Kumar K., Muller C.E., Werner J.J., Angenent LT, Bell M.E., Hay A.G., Peterson D.A., Walter J., Vijay-Kumar M., Gewirtz A.T., Ley RE. (2013) Innate and adaptive immunity interact to quench microbiome flagellar motility in the gut. Cell Host Microbe, 14, 571-581.

D'Auria G., Peris-Bondia F., Dzunkova M., Mira A., Collado M.C., Latorre A., Moya A. (2013) Active and secreted IgA-coated bacterial fractions from the human gut reveal an under-represented microbiote core. Sci Rep, 3, 3515.

Davenport E.R., Cusanovich D.A., Michelini K., Michelini K., Barreiro L.B., Ober C., Gilad Y. (2015) Genomewide association studies of the human gut microbiota. PLoS One, 10, e0140301 
Forslund K., Hildebrand F., Nielsen T., Falony G., Le Chatelier E., Sunagawa S., Prifti E., Vieira-Silva S., Gudmundsdottir V., Krogh Pedersen H., Arumugam M., Kristiansen K., Voigt A.Y., Vestergaard H., Hercog R., Igor Costea P., Kultima J.R., Li J., Jørgensen T., Levenez F., Doré J ; MetaHIT consortium, Nielsen H.B., Brunak S., Raes J , Hansen T., Wang J., Ehrlich S.D., Bork P., Pedersen O. (2015) Disentangling type 2 diabetes and metformin treatment signatures in the human gut microbiota. Nature, 528, 262-266.

Glocker E.O., Hennigs A., Nabavi M., Schäffer A.A., Woellner C., Salzer U., Pfeifer D., Veelken H., Warnatz K., Tahami F., Jamal S., Manguiat A., Rezaei N., Amirzargar A.A., Plebani A., Hannesschläger N., Gross O., Ruland J., Grimbacher B.A. (2009) Homozygous C.A.RD9 mutation in a family with susceptibility to fungal infections. $N$ Engl J Med, 361, 1727-1735.

Goodrich J.K., Davenport E.R., Beaumont M., Jackson M.A., Knight R., Ober C., Spector TD, Bell J.T., Clark A.G., Ley R.E. (2016) Genetic determinants of the gut microbiome in U.K. twins. Cell Host Microbe, 19, 731743.

Goodrich J.K., Waters J.L., Poole A.C., Sutter J.L., Koren O., Blekhman R., Beaumont M, Van Treuren W., Knight R., Bell J.T., Spector T.D., Clark A.G., Ley R.E. (2014) Human genetics shape the gut microbiome. Cell, 159, 789-799.

Horton M.W., Bodenhausen N., Beilsmith K., Meng D., Muegge B.D., Subramanian S., Vetter M.M., Vilhjálmsson B.J., Nordborg M., Gordon J.I., Bergelson J. (2014) Genome-wide association study of Arabidopsis thaliana leaf microbial community. Nat Commun, 5, 320.

Hov J.R., Zhong H., Qin B., Anmarkrud J.A., Holm K, Franke A, Lie B.A., Karlsen T.H. (2015) The influence of the autoimmunity-associated ancestral HLA haplotype AH8.1 on the human gut microbiota : a crosssectional study. PLoS One, 10, e0133804.

Hua X., Goedert J.J., Pu A., Yu G., Shi J. (2016) Allergy associations with the adult fecal microbiota : analysis of the American Gut Project. EBioMedicine, 3, 172179.

Kamada N., Seo S.U., Chen G.Y., Nunez G. (2013) Role of the gut microbiota in immunity and inflammatory disease. Nat Rev Immunol, 13, 321-335.

Kau A.L., Planer J.D., Liu J., Rao S., Yatsunenko T., Trehan I., Manary M.J., Liu T.C., Stappenbeck T.S., Maleta K.M., Ashorn P., Dewey K.G., Houpt E.R., Hsieh C.S., Gordon J.I. (2015) Functional characterization of IgA-targeted bacterial taxa from undernourished Malawian children that produce dietdependent enteropathy. Sci Transl Med, 7, 276ra224.

Kostic A.D., Howitt M.R., Garrett W.S. (2013) Exploring host-microbiota interactions in animal models and humans. Genes Dev, 27, 701-718.
Krzywinski M., Schein J., Birol I., Connors J., Gascoyne R., Horsman D., Jones S.J., Marra M.A. (2009) Circos : an information aesthetic for comparative genomics. Genome Res, 19, 1639-1645.

Kubinak J.L., Petersen C., Stephens W.Z., Soto R., Bake E., O’Connell R.M., Round J.L. (2015) MyD88 signaling in $\mathrm{T}$ cells directs IgA-mediated control of the microbiota to promote health. Cell Host Microbe, 17, 153-163.

Lamas B., Richard M.L., Leducq V., Pham H.P., Michel M.L., Da Costa G., Bridonneau C., Jégou S., Hoffmann T.W., Natividad J.M., Brot L., Taleb S., CouturierMaillard A, Nion-Larmurier I., Merabtene F., Seksik P., Bourrier A., Cosnes J., Ryffel B., Beaugerie L., Launay J.M., Langella P., Xavier R.J., Sokol H., (2016) CARD9 impacts colitis by altering gutmicrobiota metabolism of tryptophan into aryl hydrocarbon receptor ligands. Nat Med, 22, 598-605.

Lavasani S., Dzhambazov B., Nouri M., Fåk F., Buske S., Molin G., Thorlacius H, Alenfall J., Jeppsson B., Weström B. (2010) A novel probiotic mixture exerts a therapeutic effect on experimental autoimmune encephalomyelitis mediated by IL-10 producing regulatory T cells. PLoS One, 5, e9009.

Liang X., Bushman F.D., FitzGerald G.A. (2015) Rhythmicity of the intestinal microbiote is regulated by gender and the host circadian clock. Proc Natl Acad Sci USA, 112, 10479-10484.

Lim M.Y., Yoon H.S., Rho M., Sung J., Song Y.M., Lee K., Ko G. (2016a) Analysis of the association between host genetics, smoking, and sputum microbiota in healthy humans. Sci Rep, 6, 23745.

Lim M.Y., You H.J., Yoon H.S., Kwon B., Lee J.Y., Lee S., Song Y.M., Lee K., Sung J., Ko G. (2016b) The effect of heritability and host genetics on the gut microbiota and metabolic syndrome. Gut, doi :10.1136/gutjnl2015-311326.

Moor K., Fadlallah J., Toska A., Sterlin D., Balmer M.L., Macpherson A.J., Gorochov G, Larsen M., Slack E., (2016) Analysis of bacterial-surface-specific antibodies in body fluids using bacterial flow cytometry. Nat Protoc, 11, 1531-1553.

Netea M.G., Wijmenga C., O'Neill L.A. (2012) Genetic variation in Toll-like receptorsand disease susceptibility. Nat Immunol, 13, 535-542.

Ochoa-Repáraz J., Mielcarz D.W., Ditrio L.E., Burroughs A.R., Begum-Haque S., Dasgupta S., Kasper D.L., Kasper L.H. (2010) Central nervous system demyelinating disease protection by the human commensal Bacteroides fragilis depends on polysaccharide A expression. J Immunol, 185, 4101-4108.

Olivares M., Neef A., Castillejo G., Palma G.D., Varea V., Capilla A., Palau F Nova E, Marcos A., Polanco I., Ribes-Koninckx C., Ortigosa L., Izquierdo L., Sanz Y. (2015) The HLA-DQ2 genotype selects for early intestinal microbiota composition in infants at high risk of developing coeliac disease. Gut, 64, 406-417. 
Org E., Parks B.W., Joo J.W., Emert B., Schwartzman W., Kang E.Y., Mehrabian M., Pan C, Knight R., Gunsalus R., Drake T.A., Eskin E., Lusis A.J. (2015) Genetic and environmental control of host-gut microbiota interactions. Genome Res, 25, 1558-1569.

Ou J., Carbonero F., Zoetendal E.G., DeLany J.P., Wang M., Newton K., Gaskins H.R., O'Keefe S.J. (2013) Diet, microbiota, and microbial metabolites in colon cancer risk in rural Africans and African Americans. Am J Clin Nutr, 98, 111-120.

Palm N.W., de Zoete M.R., Cullen T.W., Barry N.A., Stefanowski J., Hao L., Degnan P.H., Hu J., Peter I., Zhang W., Ruggiero E., Cho J.H., Goodman A.L., Flavell R.A. (2014) Immunoglobulin A coating identifies colitogenic bacteria in inflammatory bowel disease. Cell, 158, 1000-1010.

Planer J.D., Peng Y., Kau A.L., Blanton L.V., Ndao I.M., Tarr P.I., Warner B.B., Gordon J.I. (2016) Development of the gut microbiota andmucosal $\operatorname{IgA}$ responses in twins and gnotobiotic mice. Nature, 534, $263-266$.

Portelli M.A., Hodge E., Sayers I. (2015) Genetic risk factors for the development of allergic disease identified by genome-wide association. Clin Exp Allergy, 45, 21-31.

Qin J., Li Y., Cai Z., Li S., Zhu J., Zhang F., Liang S., Zhang W., Guan Y., Shen D., Peng Y., Zhang D., Jie Z., Wu W., Qin Y., Xue W., Li J., Han L., Lu D., Wu P., Dai Y., Sun X., Li Z., Tang A., Zhong S., Li X., Chen W., Xu R., Wang M., Feng Q., Gong M., Yu J, Zhang Y., Zhang M., Hansen T., Sanchez G., Raes J., Falony G., Okuda S., Almeida M, Le Chatelier E., Renault P., Pons N., Batto J.M., Zhang Z., Chen H., Yang R., Zheng W., Li S., Yang H., Wang J., Ehrlich S.D., Nielsen R., Pedersen O., Kristiansen K., Wang J. (2012) A metagenome-wide association study of gut microbiota in type 2 diabetes. Nature, 490, 55-60.
Rochereau N., Drocourt D., Pérouzel E., Pavot V., Redelinghuys P., Brown G.D., Tiraby G., Roblin X., Verrier B., Génin C., Corthésy B., Paul S. (2013) Dectin-1 is essential for reverse transcytosis of glycosylated SIgA-antigen complexes by intestinal M cells. PLoS Biol, 11, e1001658.

Round J.L., Mazmanian S.K. (2009) The gut microbiota shapes intestinal immune responses during health and disease. Nat Rev Immunol, 9, 313-323.

Round J.L.,Mazmanian S.K. (2010) Inducible Foxp3b regulatory T-cell development by a commensal bacterium of the intestinal microbiota. Proc Natl Acad Sci USA, 107, 12204-12209.

Si J., Lee S., Park J.M., Sung J., Ko G. (2015) Genetic associations and shared environmentaleffects on the skin microbiome of Korean twins. BMC Genomics, 16, 992.

van de Merwe J.P., Stegeman J.H., Hazenberg M.P. (1983) The resident faecal flora is determined by genetic characteristics of the host. Implications for Crohn's disease? Antonie Van Leeuwenhoek, 49, 119-124.

Woon P.Y., Kaisaki P.J., Braganca J., Bihoreau M.T., Levy J.C., Farrall M., Gauguier D. (2007) Aryl hydrocarbon receptor nuclear translocator-like (BMAL1) is associated with susceptibility to hypertension and type 2 diabetes. Proc Natl Acad Sci USA, 104, 14412-14417.

Yatsunenko T., Rey F.E., Manary M.J., Trehan I., Dominguez-Bello M.G., Contreras M., Magris M., Hidalgo G., Baldassano R.N., Anokhin A.P., Heath A.C., Warner B., Reeder J, Kuczynski J., Caporaso J.G., Lozupone C.A., Lauber C., Clemente J.C., Knights D., Knight R., Gordon J.I. (2012) Human gut microbiome viewed across age and geography. Nature, 486, 222-227. 